\title{
Perineural invasion as a prognostic risk factor in patients with early cervical cancer
}

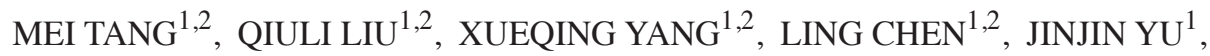 \\ XIAOWEI QI ${ }^{1}$ and YUAN WANG ${ }^{1}$ \\ ${ }^{1}$ Department of Obstetrics and Gynecology, Affiliated Hospital of Jiangnan University, Wuxi, Jiangsu 214062; \\ ${ }^{2}$ Department of Medicine, Jiangnan University, Wuxi, Jiangsu 214000, P.R. China
}

Received December 6, 2017; Accepted September 28, 2018

DOI: $10.3892 / \mathrm{ol} .2018 .9674$

\begin{abstract}
The aim of the present study was to investigate the influence of perineural invasion (PNI) on the prognosis of patients with early cervical cancer (stages IA2-IIA2). A retrospective analysis was conducted on 406 patients with early cervical cancer who underwent a radical hysterectomy and pelvic lymphadenectomy between January 2007 and December 2014 at the Affiliated Hospital of Jiangnan University (Wuxi, China). The clinicopathological data of the patients were obtained and follow-up assessments were performed. A statistical analysis of the association between PNI and each index was performed, and the effect of PNI and the clinicopathological parameters on the prognosis of the patients was evaluated. Among the 406 cases with early cervical cancer, 41 cases were lost, with a follow-up rate of $89.90 \%$. Overall, 43 PNI-positive patients were observed, with an occurrence rate of $10.59 \%$. PNI-positivity was associated with hypertension, lymph node metastasis, depth of cervical invasion, surgical margin and vascular invasion $(\mathrm{P}<0.05)$, but it was not associated with age, diabetes, clinical stage, histological type or tumor size $(\mathrm{P}>0.05)$. The overall survival (OS) and disease-free survival (DFS) times of PNI-positive patients were significantly lower compared with those of PNI-negative patients. A multivariate regression analysis revealed that age, tumor size, clinical stage and PNI were independent risk factors for OS and DFS times. PNI is a poor prognostic factor for patients with early cervical cancer.
\end{abstract}

Correspondence to: Dr Yuan Wang, Department of Obstetrics and Gynecology, Affiliated Hospital of Jiangnan University, 200 Huihe Road, Wuxi, Jiangsu 214062, P.R. China

E-mail: 13915355849@163.com

Abbreviations: PNI, perineural invasion; OS, overall survival; DFS, disease-free survival; LVSI, lymph vascular space invasion; NGF, nerve growth factor; BDNF, brain-derived nerve growth factor

Key words: cervical cancer, perineural invasion, risk factors, prognosis, overall survival time, disease-free survival time

\section{Introduction}

One of the most common gynecological malignancies is cervical cancer, with its incidence ranking fourth among malignant tumors globally, and it accounts for $85 \%$ of cancer cases in developing countries; it is also the primary cause of cancer-associated mortalities in these areas (1). Although the methods of prevention and treatment of cervical cancer have improved proportionally with the improvement of the overall standard of medical practice in recent years, cervical cancer remains a major worldwide health problem. At present, the primary treatment of patients with early cervical cancer [stages IA2-IIA2, classified according to the International Federation of Gynecology and Obstetrics (2)] is to perform a radical hysterectomy and a pelvic lymphadenectomy, and if any pathological risk factors are noted postoperatively, follow-up treatment is required. Malignant tumors are primarily spread by direct spread, hematogenous metastasis, lymph node metastasis and implantation. A previous study has suggested that perineural invasion (PNI) is another independent way of transmission into the lymphatic and circulatory systems (3), and no lymphatic vessel tissue exists in the epineurium, therefore tumor PNI is not equivalent to lymph vascular space invasion (LVSI), which is another pathological feature independent of LVSI (4). As early as 1835, Cruveilheir (5) first reported the PNI phenomenon, as manifested by malignant tumors, and identified that head cancer and neck cancer can easily penetrate into the nerves and form growths in the brain. In subsequent studies, PNI was demonstrated in a number of malignant tumors, including pancreatic cancer, colorectal cancer, bladder cancer, prostate cancer and cholangiocarcinoma, and it was revealed to negatively affect patient prognosis $(6,7)$. Although there are relatively few studies on PNI in cervical cancer, one study has revealed an association between PNI and the prognosis of patients with early cervical cancer, with the 5-year overall survival (OS) time for patients with PNI-positive tumors being significantly decreased (8), and this may be a prognostic factor for these patients. On the other hand, another study has indicated that the prognosis of patients with cervical cancer was not affected by PNI, and that PNI was not associated with recurrence and survival rate (9). Therefore, whether PNI-positivity affects the prognosis of patients with cervical cancer remains unclear. The aim of the present study 
was to investigate the association between PNI and the clinical features and pathological parameters of patients with cervical cancer, to analyze the effect of PNI on the survival time of the patients, to assess whether PNI-positivity affects the prognosis of patients with early cervical cancer, and to provide a basis for the patient to select between nerve-sparing radical hysterectomy or adjuvant therapy.

\section{Patients and methods}

Patient characteristics and clinical pathology. Overall, 406 patients with early cervical cancer who underwent radical hysterectomy and pelvic lymphadenectomy between January 2007 and December 2014 at the Affiliated Hospital of Jiangnan University (Wuxi, China), were included in the present study. The patients had not received any treatment prior to surgery. A radical hysterectomy is the basic surgical procedure for treating cases of cervical cancer. At the Affiliated Hospital of Jiangnan University, the type of surgery selected for each patient depends on the clinical stage of the cancer, classified according to the Querleu-Morrow classification system (10): Stage IA1, hysterectomy outside the anadesma; Stage IA2, expanded hysterectomy, increasing 1-2 cm; Stage IB or IIA, radical hysterectomy with excision of $3 \mathrm{~cm}$ parametrium and $3 \mathrm{~cm}$ vagina. Pelvic lymph node dissection was performed according to the Querleu-Morrow guidelines. The inclusion criteria for the patients in the present study were: i) Diagnosis with cervical cancer by two senior pathologists; ii) stage IA2-IIA2 cervical cancer, classified according to the International Federation of Gynecology and Obstetrics (2009) (2); iii) a radical hysterectomy and pelvic lymphadenectomy as a method of treatment; and iv) availability of the clinical and pathological data. The recorded clinicopathological parameters included the age of onset, evidence of hypertension or diabetes, clinical stage, histological type, tumor size, lymph node metastasis, depth of cervical invasion, surgical margin, vascular invasion and PNI.

Research methods. The diagnosis and the recording of the clinical and pathological features of the patients and samples were provided by the Department of Pathology of the Affiliated Hospital of Jiangnan University. Tumor tissue samples were taken from the cervix of the patients during surgery and were fixed with $4 \%$ paraformaldehyde at $4{ }^{\circ} \mathrm{C}$ for $24 \mathrm{~h}$ and paraffin-embedded. Tissue sections were stained with hematoxylin and eosin as previously described (11), and the sections were evaluated by two senior pathologists using a light microscope. In the cases of differing evaluations, discrepancies were settled by the more senior pathologist. The present study focused on PNI in the cervix, and not on parametrial invasion. The definition of PNI-positivity (12) was based on the location of the tumor cells around the nerve fibers, with the cells being present along the nerve into the epineurium, perineurium or endoneurium of any layer, or gathered and wrapped around the nerve for $\geq 33 \%$ of its diameter and spread along the expansion of the tumor cells with local infiltration. All 406 patients were followed up by telephone every 3 months between the time of diagnosis and January 16, 2017, until the occurrence of tumor recurrence
Table I. Association between PNI and clinical and pathological features.

\begin{tabular}{|c|c|c|c|}
\hline Parameter & PNI-negative & PNI-positive & P-value \\
\hline Age, years & $48.59 \pm 9.61$ & $47.37 \pm 9.27$ & 0.118 \\
\hline Hypertension & & & $<0.001$ \\
\hline Negative & $325(89.5 \%)$ & $30(69.8 \%)$ & \\
\hline Positive & $38(10.5 \%)$ & $13(30.2)$ & \\
\hline Diabetes & & & 0.093 \\
\hline Negative & $344(94.8 \%)$ & $38(88.4 \%)$ & \\
\hline Positive & $19(5.2 \%)$ & $5(11.6 \%)$ & \\
\hline Clinical stage & & & 0.148 \\
\hline IA 2 & $7(1.9 \%)$ & $1(2.3 \%)$ & \\
\hline IB 1 & $206(56.7 \%)$ & $16(37.2 \%)$ & \\
\hline IB2 & $41(11.3 \%)$ & $9(20.9 \%)$ & \\
\hline IIA1 & $68(18.7 \%)$ & $11(25.6 \%)$ & \\
\hline Histological type & & & 0.357 \\
\hline $\begin{array}{l}\text { Squamous cell } \\
\text { carcinoma }\end{array}$ & $321(89.0 \%)$ & $41(95.3 \%)$ & \\
\hline Adenocarcinoma & $31(8.5 \%)$ & $1(2.3 \%)$ & \\
\hline $\begin{array}{l}\text { Adenosquamous } \\
\text { carcinoma }\end{array}$ & $9(2.5 \%)$ & $1(2.3 \%)$ & \\
\hline Tumor size, $\mathrm{cm}$ & & & 0.560 \\
\hline$\leq 4$ & $244(67.2 \%)$ & $27(62.8 \%)$ & \\
\hline$>4$ & $119(32.8 \%)$ & $16(37.2 \%)$ & \\
\hline $\begin{array}{l}\text { Lymph node } \\
\text { metastasis }\end{array}$ & & & $<0.001$ \\
\hline Negative & $296(81.5 \%)$ & $21(48.8 \%)$ & \\
\hline Positive & $67(18.5 \%)$ & $22(51.2 \%)$ & \\
\hline $\begin{array}{l}\text { Depth of cervical } \\
\text { invasion }\end{array}$ & & & $<0.001$ \\
\hline$<2 / 3$ & $171(47.1 \%)$ & $6(14.0 \%)$ & \\
\hline$\geq 2 / 3$ & $192(52.9 \%)$ & $37(86.0 \%)$ & \\
\hline Surgical margin & & & 0.002 \\
\hline Negative & $362(99.7 \%)$ & $41(95.3 \%)$ & \\
\hline Positive & $1(0.3 \%)$ & $2(4.7 \%)$ & \\
\hline Vascular invasion & & & $<0.001$ \\
\hline Negative & $251(69.1 \%)$ & $15(34.9 \%)$ & \\
\hline Positive & $112(30.9 \%)$ & $28(65.1 \%)$ & \\
\hline
\end{tabular}

PNI, perineural invasion.

or mortality. The follow-up content included: i) The general condition of the patient; ii) questions of whether to have the regular follow-up, the results of the follow-up and the last follow-up time; iii) the timing of any recurrence or metastasis, and iv) the patients who succumbed, so as to understand the reasons for tumor recurrence and mortality. Prognostic indicators included the OS time, defined as the time between the date of surgery or diagnosis and the time of patient mortality or last follow-up, and the disease-free survival (DFS) time, defined as the time between the date of surgery and the time of the first recurrence in the patient or last follow-up. 

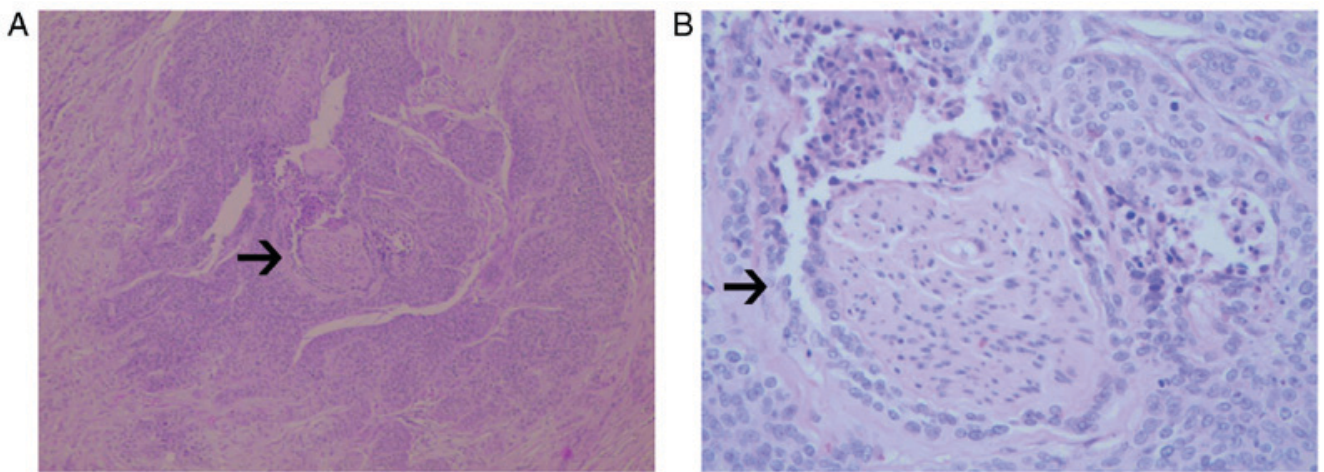

Figure 1. Photomicrographs of the histology of perineural invasion (arrows) in cervical cancer tissue, following hematoxylin and eosin staining, at (A) low-power (x100) and (B) high-power magnifications (x200) of tumor cells wrapping around and invading the nerve.
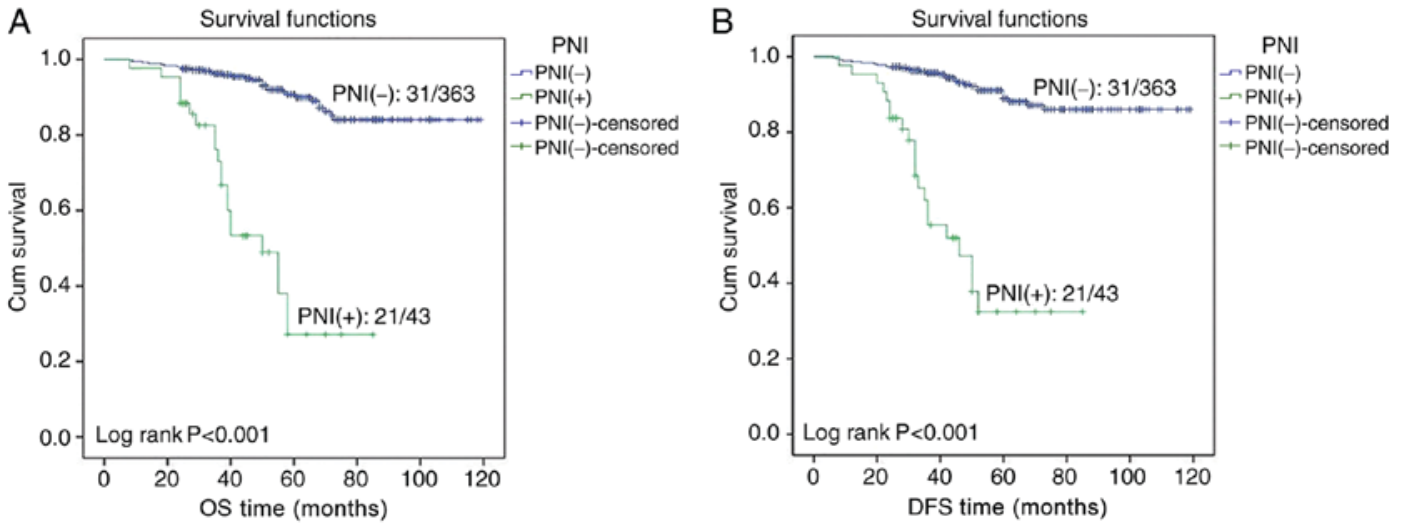

Figure 2. Effect of PNI on the survival times of patients with early cervical cancer. (A) Kaplan-Meier curve for OS rate for PNI-positive and -negative cases. (B) Kaplan-Meier curve for DFS rate for PNI-positive and -negative cases. PNI, perineural invasion; Cum, cumulative; OS, overall survival; DFS, disease-free survival.

Statistical analysis. The statistical analyses of the data was performed using the IBM SPSS Statistics software (version 20.0; IBM Corp., Armonk, NY, USA). The associations between PNI and other clinical features and pathological indices were analyzed using a $\chi^{2}$ test; a Kaplan-Meier analysis was performed to calculate and compare the survival rate of the patients; the log-rank test was used to determine whether the survival curve showed significant differences; and a Cox proportional hazards regression model was used to analyze the influence of the clinical characteristics and pathological features on the patient prognosis.

\section{Results}

Association between PNI and clinicopathological features of patients with early cervical cancer. Of the 406 cases of early cervical cancer included in the present study, 41 cases were lost, with a follow-up rate of $89.90 \%$. The incidence of PNI was $43 / 406$ (10.59\%). With respect to microscopic observations, in a number of the PNI-positive cases there was a manifestation of tumor cells around the nerve and nerve fibers, and local invasion and metastasis along its extension (Fig. 1). The average age of the 363 patients without PNI was $48.59 \pm 9.61$ years and that of the 43 patients with PNI was $47.37 \pm 9.27$ years $(\mathrm{P}=0.118)$. The phenomenon of $\mathrm{PNI}$ was significantly associated with hypertension $(\mathrm{P}<0.001)$, but there was no association between PNI and diabetes $(\mathrm{P}=0.093)$ or clinical stage $(\mathrm{P}=0.148)$. PNI was significantly associated with lymph node metastasis $(\mathrm{P}<0.001)$, depth of cervical invasion $(\mathrm{P}<0.001)$, surgical margin $(\mathrm{P}=0.002)$, and vascular invasion $(\mathrm{P}<0.001)$, but not with tumor histological type $(\mathrm{P}=0.357)$ or size $(\mathrm{P}=0.560)$ (Table $\mathrm{I})$. These results indicate that PNI is associated with risk factors that have an effect on the prognosis of patients with cervical cancer.

Effect of PNI on survival times of patients with early cervical cancer. In the patients with early cervical cancer, Kaplan-Meier survival curves indicated that the OS and DFS times of PNI-positive patients were significantly lower compared with those of patients without PNI (both $\mathrm{P}<0.001$; Fig. 2). In the 2017 National Comprehensive Cancer Network clinical practice guidelines (1), tumor size and lymph node metastasis are risk factors for patients with cervical cancer. Therefore, tumor size and lymph node metastasis were selected for further survival analysis. A survival analysis was performed for patients with tumors of size $\leq 4$ or $>4 \mathrm{~cm}$, and the Kaplan-Meier curves revealed that the OS and DFS times in PNI-positive patients were significantly lower compared with in PNI-negative patients for both categories of tumor size (all $\mathrm{P}<0.001$; Fig. 3). A survival analysis was also performed for patients with or without lymph node metastasis, and the Kaplan-Meier curves demonstrated that the OS and DFS times were significantly less for PNI-positive compared with 


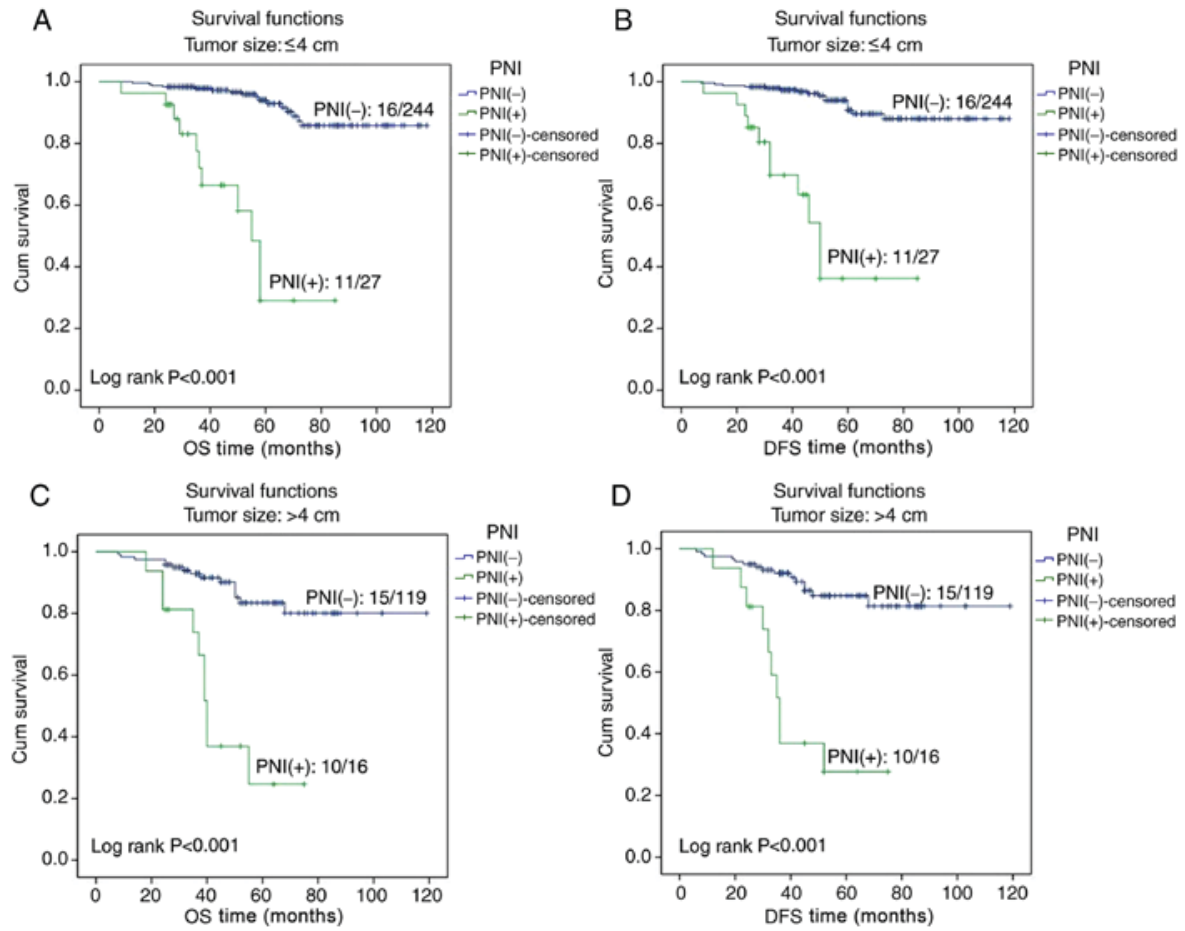

Figure 3. Effect of PNI on the survival times of patients with tumor size $\leq 4$ or $>4 \mathrm{~cm}$. The Kaplan-Meier curves for (A) OS time of patients with tumor size $\leq 4 \mathrm{~cm}$ with and without PNI, (B) DFS time of patients with tumor size $\leq 4 \mathrm{~cm}$ with and without PNI, (C) OS time of patients of tumor size $>4 \mathrm{~cm}$ with and without PNI, and (D) DFS time of patients with tumor size $>4 \mathrm{~cm}$ with and without PNI. PNI, perineural invasion; OS, overall survival; DFS, disease-free survival; Cum, cumulative.
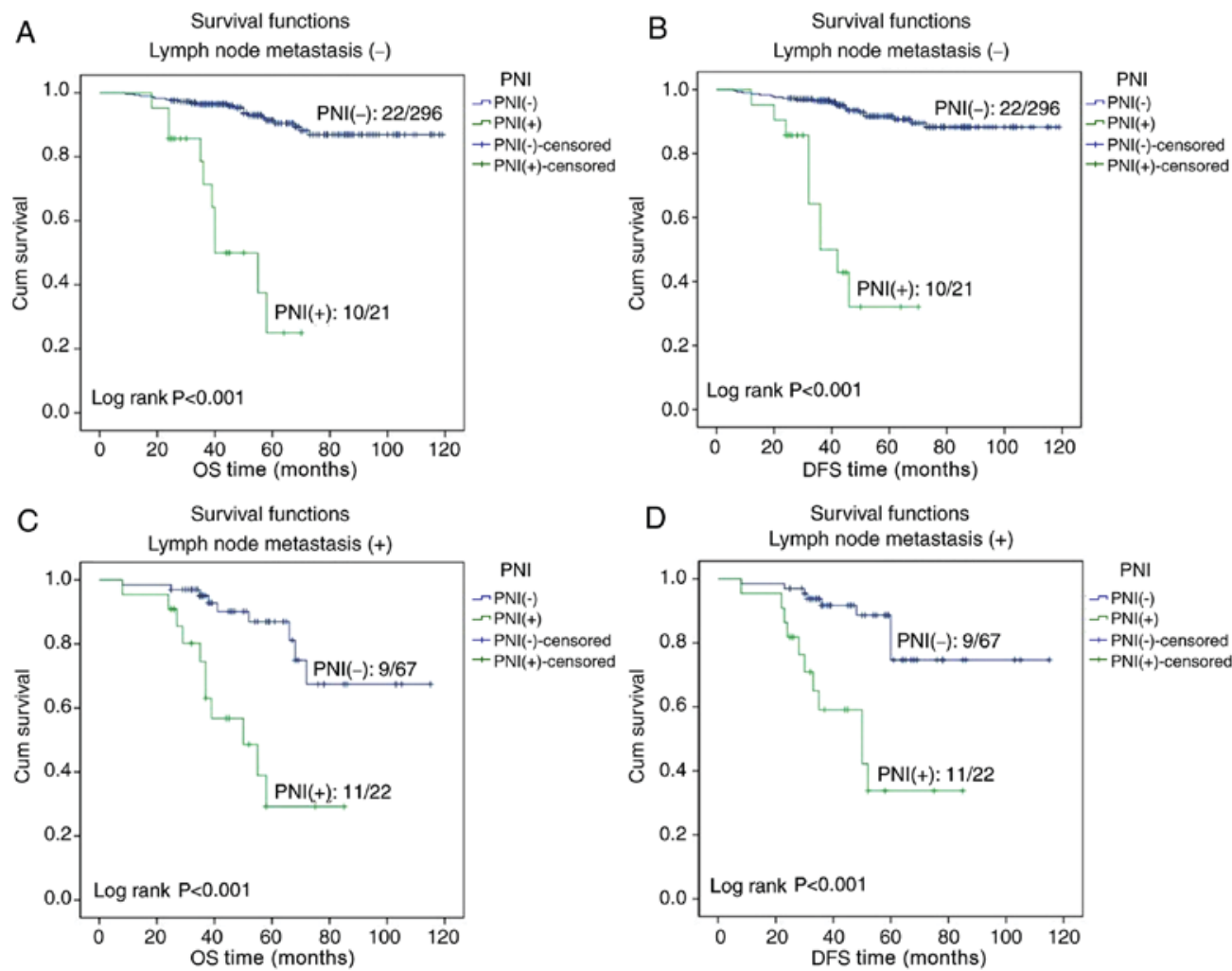

Figure 4. Effect of PNI on the survival time of patients with or without lymph node metastasis. The Kaplan-Meier curves for (A) OS time of patients without lymph node metastasis with and without PNI, (B) DFS time of patients without lymph node metastasis with and without PNI, (C) OS time of patients with lymph node metastasis with and without PNI, and (D) DFS time of patients with lymph node metastasis with and without PNI. PNI, perineural invasion; OS, overall survival; DFS, disease-free survival; Cum, cumulative.

PNI-negative patients, regardless of the lymph node metastasis status (all $\mathrm{P}<0.001$; Fig. 4).
A Kaplan-Meier survival analysis was used to calculate the 3- and 5-year survival rates of patients, and revealed that the 
Table II. Univariate and multivariate survival analyses for independent risk factors for overall survival time.

\begin{tabular}{|c|c|c|c|c|c|c|}
\hline \multirow[b]{2}{*}{ Parameter } & \multicolumn{2}{|c|}{ Univariate analysis } & \multirow[b]{2}{*}{ P-value } & \multicolumn{2}{|c|}{ Multivariate analysis } & \multirow[b]{2}{*}{ P-value } \\
\hline & $\mathrm{RR}$ & $95 \% \mathrm{CI}$ & & RR & $95 \% \mathrm{CI}$ & \\
\hline Age & 1.024 & $0.996-1.053$ & 0.093 & 1.047 & $1.015-1.081$ & 0.004 \\
\hline Hypertension & 1.328 & $0.625-2.821$ & 0.461 & 0.698 & $0.290-1.680$ & 0.423 \\
\hline Diabetes & 0.830 & $0.258-2.664$ & 0.754 & 0.417 & $0.121-1.437$ & 0.166 \\
\hline Clinical stage & 0.923 & $0.717-1.187$ & 0.531 & 0.653 & $0.479-0.890$ & 0.007 \\
\hline Histological type & 1.314 & $0.743-2.322$ & 0.347 & 1.553 & $0.847-2.849$ & 0.155 \\
\hline Tumor size & 2.144 & $1.244-3.698$ & 0.006 & 2.832 & $1.538-5.218$ & 0.001 \\
\hline Lymph node metastasis & 2.636 & $1.505-4.615$ & 0.001 & 1.602 & $0.862-2.977$ & 0.136 \\
\hline Depth of cervical invasion & 3.149 & $1.123-8.831$ & 0.029 & 0.638 & $0.338-1.206$ & 0.167 \\
\hline Surgical margin & 7.431 & $0.998-55.354$ & 0.050 & 1.789 & $0.227-14.071$ & 0.580 \\
\hline Vascular invasion & 1.390 & $0.798-2.419$ & 0.244 & 0.762 & $0.401-1.446$ & 0.406 \\
\hline PNI & 9.267 & $5.266-16.307$ & $<0.001$ & 14.621 & $6.974-30.652$ & $<0.001$ \\
\hline
\end{tabular}

RR, relative risk; CI, confidence interval, PNI, perineural invasion.

Table III. Univariate and multivariate survival analyses for independent risk factor for disease-free survival time.

\begin{tabular}{|c|c|c|c|c|c|c|}
\hline \multirow[b]{2}{*}{ Parameter } & \multicolumn{2}{|c|}{ Univariate analysis } & \multirow[b]{2}{*}{ P-value } & \multicolumn{2}{|c|}{ Multivariate analysis } & \multirow[b]{2}{*}{ P-value } \\
\hline & $\mathrm{RR}$ & $95 \% \mathrm{CI}$ & & $\mathrm{RR}$ & $95 \% \mathrm{CI}$ & \\
\hline Age & 1.025 & $0.996-1.054$ & 0.089 & 1.047 & $1.014-1.081$ & 0.005 \\
\hline Hypertension & 1.344 & $0.633-2.855$ & 0.442 & 0.693 & $0.288-1.664$ & 0.411 \\
\hline Diabetes & 0.839 & $0.261-2.695$ & 0.768 & 0.386 & $0.111-1.345$ & 0.135 \\
\hline Clinical stage & 0.927 & $0.720-1.192$ & 0.553 & 0.654 & $0.478-0.895$ & 0.008 \\
\hline Histological type & 1.285 & $0.724-2.282$ & 0.392 & 1.521 & $0.826-2.803$ & 0.178 \\
\hline Tumor size & 2.084 & $1.209-3.592$ & 0.008 & 2.734 & $1.479-5.052$ & 0.001 \\
\hline Lymph node metastasis & 2.592 & $1.481-4.539$ & 0.001 & 1.634 & $0.880-3.034$ & 0.120 \\
\hline Depth of cervical invasion & 3.237 & $1.149-9.125$ & 0.026 & 0.633 & $0.336-1.192$ & 0.157 \\
\hline Surgical margin & 6.227 & $0.845-45.897$ & 0.073 & 1.455 & $0.188-11.273$ & 0.720 \\
\hline Vascular invasion & 1.393 & $0.800-2.424$ & 0.242 & 0.746 & $0.395-1.411$ & 0.368 \\
\hline PNI & 9.273 & $5.269-16.321$ & $<0.001$ & 14.923 & 7.147-31.162 & $<0.001$ \\
\hline
\end{tabular}

RR, relative risk; CI, confidence interval; PNI, perineural invasion.

3- and 5-year OS rate for patients with PNI were significantly lower compared with for patients without PNI (73.0 vs. 95.9, and 27.2 vs. $90.0 \%$, respectively; $\mathrm{P}<0.001$ ); the 3 - and 5-year DFS rates for patients with PNI were also significantly less compared with for patients without PNI (55.5 vs. 95.7, and 32.4 vs. $88.9 \%$, respectively; $\mathrm{P}<0.001)$.

Univariate and multivariate analyses of prognosis. The univariate Cox regression analysis revealed that the OS time in patients with early cervical cancer was significantly associated with tumor size $(\mathrm{P}=0.006)$, lymph node metastasis $(\mathrm{P}=0.001)$, depth of cervical invasion $(\mathrm{P}=0.029)$ and PNI $(\mathrm{P}<0.001)$, but was not associated with age $(\mathrm{P}=0.093)$, hypertension $(\mathrm{P}=0.461)$, diabetes $(\mathrm{P}=0.754)$, clinical stage $(\mathrm{P}=0.531)$, histological type $(\mathrm{P}=0.347)$, surgical margin
$(\mathrm{P}=0.050)$ or vascular invasion $(\mathrm{P}=0.244)$. The multivariate Cox regression analysis revealed that age, clinical stage, tumor size and PNI were independent risk factors for the OS time $(\mathrm{P}=0.004, \mathrm{P}=0.007, \mathrm{P}=0.001, \mathrm{P}<0.001$, respectively) (Table II).

With regard to the DFS time in these patients, the univariate analysis revealed that it was significantly associated with tumor size $(\mathrm{P}=0.008)$, lymph node metastasis $(\mathrm{P}=0.001)$, depth of cervical invasion $(\mathrm{P}=0.026)$ and $\mathrm{PNI}(\mathrm{P}<0.001)$, but not with age $(\mathrm{P}=0.089)$, hypertension $(\mathrm{P}=0.442)$, diabetes $(\mathrm{P}=0.768)$, clinical stage $(\mathrm{P}=0.553)$, histological type $(\mathrm{P}=0.392)$, surgical margin $(\mathrm{P}=0.073)$ or vascular invasion $(\mathrm{P}=0.242)$. The multivariate analysis revealed that age, clinical stage, tumor size and PNI were independent risk factors for the DFS time $(\mathrm{P}=0.005$, $\mathrm{P}=0.008, \mathrm{P}=0.001$ and $\mathrm{P}<0.001$, respectively) (Table III). 


\section{Discussion}

Around the nerve fibers, tumor cells can invade into the epineurium, perineurium or endoneurium, and the phenomenon of local invasion and metastasis along its extension is known as PNI. The pathological characteristics of PNI are the existence of tumor cells close to the nerve and surrounding $>33 \%$ of the nerve circumference, or in any layer of epineurium, perineurium or endoneurium; this is defined as PNI-positivity (13).

A previous study has suggested that axonal migration is a key factor in the occurrence of PNI; nerve growth factor (NGF), brain-derived nerve growth factor (BDNF), and neurotropic factors 3 and 4 are all neurotropins that are important in the process of axonal growth (14). The two primary NGF receptors are the neurotrophic receptor tyrosine kinase (Trk) family (containing high-affinity receptors), and the tumor necrosis factor receptor p75 (low-affinity). A number of studies have demonstrated that the expression of NGF and its receptors TrkA and p75 in pancreatic cancer cells and their peripheral nerve cells increased, indicating that the expression of NGF and its receptors TrkA and p75 in these tissues is associated with the occurrence of PNI $(15,16)$. Regarding other nerve growth factors, an increase in the expression of BDNF can promote the occurrence of PNI, and the proliferation and invasion of tumor cells are associated with the high expression of BDNF (12). TrkB is a BDNF receptor in metastatic pancreatic ductal carcinoma, and an increase in its expression is associated with the presence of PNI (12). A number of malignant tumors possess a mechanism that involves PNI, but the number of studies on the molecular mechanisms associated with PNI and cervical cancer is relatively small, such that the phenomenon requires further study.

Controversy remains as to whether PNI may be used as a prognostic indicator of cervical cancer. Memarzadeh et al (17) first conducted a clinical study on PNI in cervical cancer parametrial tissue, and the results revealed that high-risk factors for recurrence include large tumors $(>4 \mathrm{~cm})$, PNI in the parametrial tissue, depth of cervical matrix infiltration $(>2 / 3)$ and lymphatic vessel invasion, whereas survival regression analysis demonstrated that PNI can be a prognostic risk factor for patients with early cervical cancer. Ozan et al (18) reported results on $36 \mathrm{IBl} / \mathrm{IB} 2$ stage postoperative cervical cancer cases, and revealed that the recurrence and mortality rates of the patients was not associated with PNI in the uterus. However, a $\chi^{2}$ analysis demonstrated that PNI was associated with risk factors such as vaginal and lymphatic invasion, suggesting that PNI is an important factor affecting the prognosis of patients with cervical cancer. A clinical study on PNI in patients with cervical cancer indicated that the 5-year OS time in PNI-positive patients was significantly decreased (8). The clinical stage of the tumor, pelvic lymph node positivity, tumor grade and PNI were all revealed to be independent prognostic factors affecting the OS time. However, ElSahwi et al (9) recorded the pathological characteristics of 192 patients with cervical cancer and revealed that PNI was associated with the tumor size and staging, but not with the recurrence or the survival rate. In 2013, Cho et al (19) used a retrospective analysis to evaluate whether PNI can be used as a risk factor for selecting an adjuvant therapy, and demonstrated that the occurrence of lymph node metastasis in PNI-positive patients was significantly increased, and that the 5-year survival rate was relatively lower in PNI-positive patients; this implied that PNI is likely to be a novel risk factor for predicting cancer recurrence. The results of the present study indicated that the occurrence of PNI is associated with hypertension, lymph node metastasis, depth of cervical invasion, surgical margin and vascular invasion, suggesting that PNI is associated with the risk factors that affect prognosis. The OS and DFS time of patients with PNI were significantly decreased compared with those of patients without PNI, indicating that PNI-positivity affects the survival rate of the patient. Univariate and multivariate survival analyses revealed that PNI is an important independent prognostic factor, whereas the multivariate analysis indicated that age, clinical stage and tumor size are also independent risk factors for OS and DFS times. Collectively, these results suggest that the aforementioned factors should be regarded more seriously.

If there is a high-risk pathological factor, including lymph node metastasis, surgical margin, and parametrial invasion, patients with early cervical cancer are advised to seek follow-up treatment. Any case presenting with a high-risk factor is recommended for postoperative pelvic irradiation with simultaneous chemotherapy of cisplatin, and brachytherapy of the vagina. At present, it is generally accepted that a parametrial invasion is an indicator of poor prognosis, and its pathological description necessitates treatment, but the existence of PNI is often ignored. A number of studies have indicated that PNI is associated with certain risk factors, as PNI-positive patients have a relatively poor prognosis, indicating that PNI is a prognostic factor for decreased survival time $(8,18,19)$. However, PNI, as a novel risk factor for cervical cancer, may provide a guiding influence as to whether postoperative patients should select adjuvant therapy. Therefore, further studies on PNI are required, investigating its mechanism of action and clarifying its prognostic significance, so as to allow it to receive the appropriate attention in order to decrease the recurrence and mortality rates in cervical cancer, and improve the DFS and OS times of the patients.

\section{Acknowledgements}

Not applicable.

Funding

The present study was supported by grants from the Maternal and Child Health Research Project of Wuxi Municipal Health and Family Planning Commission (grant no. FYKT201703) and the Wuxi Key Medical Talents Cultivation Project (grant no. ZDRCPY014).

\section{Availability of data and materials}

All data generated or analyzed during the present study are included in this published article.

\section{Authors' contributions}

YW and MT designed the study. MT designed the experiments and performed the majority of them. QL and JY were 
responsible for patient's follow-up. LC and XY collected clinical samples. XQ and JY evaluated the tissue sections. MT and YW wrote the manuscript. All authors read and approved the manuscript.

\section{Ethics approval and consent to participate}

All participants provided written informed consent prior to participating in the study. The study was approved by the Ethical Committee of the Affiliated Hospital of Jiangnan University.

\section{Patient consent for publication}

Written informed consent was obtained from all patients for the publication of their data.

\section{Competing interests}

The authors declare that they have no competing interests.

\section{References}

1. Zhou H, Liu Z, Lin ZQ: 2017 NCCN Cervical Cancer Clinical Practice Guideline Interpretation. Chin J Practical Gynecol Obstetrics 1: 100-107, 2017 (In Chinese).

2. Pecorelli S: Revised FIGO staging for carcinoma of the vulva, cervix, and endometrium. Int J Gynecol Obstet 105: 103-104, 2009.

3. Hassan MO and Maksem J: The prostatic perineural space and its relation to tumor spread: An ultrastructural study. Am J Surg Pathol 4: 143-148, 1980 .

4. Olsson Y: Microenvironment of the peripheral nervous system under normal and pathological conditions. Crit Rev Neurobiol 5: 265-311, 1990

5. Cruveilheir J: Maladies des nerfs anatomic pathologique ducorps humain 2nd edition. Paris, JB Bailliere: 3-8, 1835 (In French).

6. Cui L, Shi Y and Zhang GN: Perineural invasion as a prognostic factor for cervical cancer: A systematic review and meta-analysis. Arch Gynecol Obstet 292: 13-19, 2015.
7. Zhang GN and Cui L: Cervical cancer peripheral nerve invasion: Should pay attention to the pathological prognostic factors. Cancer Prevention Treatment 26: 183-186, 2013.

8. Horn LC, Meinel A, Fischer U, Bilek K and Hentschel B: Perineural invasion in carcinoma of the cervix uteri-prognostic impact. J Cancer Res Clin Oncol 136: 1557-1562, 2010.

9. ElSahwi KS, Barber E, Illuzzi J, Buza N, Ratner E, Silasi DA, Santin AD, Azodi M, Schwartz PE and Rutherford TJ: The significance of perineural invasion in early-stage cervical cancer. Gynecol Oncol 123: 561-564, 2011.

10. Querleu D, Cibula D and Abu-Rustum NR: 2017 Update on the Querleu-morrow classification of radical hysterectomy. Ann Surg Oncol 24: 3406-3412, 2017.

11. Luna LG: Hematoxylin and eosin staining problems and solutions. J Histotechnol 6: 16, 2013

12. Liebig C, Ayala G, Wilks JA, Berger DH and Albo D: Perineural invasion in cancer: A review of the literature. Cancer 115: 3379-3391, 2009

13. Marchesi F, Piemonti L, Mantovani A and Allavena P: Molecular mechanisms of perineural invasion, a forgotten pathway of dissemination and metastasis. Cytokine Growth Factor Rev 21: 77-82, 2010.

14. Liebig C, Ayala G, Wilks J, Verstovsek G, Liu H, Agarwal N, Berger DH and Albo D: Perineural invasion is an independent predictor of outcome in colorectal cancer. J Clin Oncol 27: 5131-5137, 2009.

15. Zhang Y, Dang C, Ma Q, Chen W and Nagata K: Predictors of systemic chemotherapy contraindication in pancreatic cancer patients with distant metastasis. Hepatogastroenterology 54: 254-259, 2007.

16. Wood JN: Nerve growth factor and pain. N Engl J Med 363: $1572-1573,2010$

17. Memarzadeh S, Natarajan S, Dandade DP, Ostrzega N, Saber PA Busuttil A, Lentz SE and Berek JS: Lymphovascular and perineural invasion in the parametria: A prognostic factor for early-stage cervical cancer. Obstet Gynecol 102: 612-619, 2003.

18. Ozan H, Ozuysal S and Ediz B: Perineural invasion in early-stage cervical carcinoma. Eur J Gynaecol Oncol 30: 379-383, 2009.

19. Cho HC, Kim H, Cho HY, Kim K, No JH and Kim YB Prognostic significance of perineural invasion in cervical cancer. Int J Gynecol Pathol 32: 228-233, 2013.

(i) (5) This work is licensed under a Creative Common Attribution-NonCommercial-NoDerivatives 4.0 International (CC BY-NC-ND 4.0) License. 